\title{
Tracking Navigation Imaging of Transcatheter Arterial Chemoembolization for Hepatocellular Carcinoma Using Three-Dimensional Cone-Beam CT Angiography
}

\author{
Yasunori Minamia ${ }^{a}$ Yukinobu Yagyu ${ }^{b} \quad$ Takamichi Murakamib $^{b}$ \\ Masatoshi Kudoa \\ aDepartment of Gastroenterology and Hepatology, and bepartment of Diagnostic Radiology, \\ Kinki University Faculty of Medicine, Osaka, Japan
}

\section{Key Words}

Feeding arteries - Hepatocellular carcinoma .

Three-dimensional cone-beam CT angiography · Tracking navigation imaging ·

Transcatheter arterial chemoembolization

\begin{abstract}
Purpose: New tracking navigation imaging software was used to evaluate the usefulness of three dimensional (3D) CT angiography for transcatheter arterial chemoembolization (TACE) in patients with hepatocellular carcinoma (HCC). Materials and Methods: Fifty-two patients with 73 HCCs were enrolled in this study retrospectively. Rotational angiography was performed from the hepatic artery for evaluation of the tumor feeding vessels. Arteries feeding the tumor were traced automatically by adjusting the region of interest around the targeted tumor on axial and coronal images using tracking navigation imaging with 3D cone-beam CT angiography. Results: Using final selective angiographic findings as the gold standard, the detection of feeding vessels was $90.4 \%(66 / 73)$ for tracking navigation imaging and $50.7 \%$ (37/73) for celiac trunk angiography. This difference was statistically significant (Wilcoxon rank sum test, $\mathrm{p}<0.001)$. The sensitivity, specificity, positive predictive value, and negative predictive value for the detection of feeding arteries were $97.1 \%(66 / 68), 80.0 \%(4 / 5), 98.5 \%$ (66/67), and $66.7 \%(4 / 6)$, respectively. The kappa coefficient had a value of 0.638 (95\% CI: $0.471-0.805)$, which is considered to indicate a good degree of agreement. With the as-
\end{abstract}

Yasunori Minami, MD, PhD

Department of Gastroenterology and Hepatology,

Kinki University Faculty of Medicine

377-2 Ohno-Higashi, Osaka-Sayama, Osaka 589-8511 (Japan)

Tel. +81 723660221 (Ext. 3149), E-Mail minkun@med.kindai.ac.jp 
sistance of tracking navigation imaging, the disease control rate of TACE for HCC was $67.3 \%$ (35/52) according to the modified Response Evaluation Criteria in Solid Tumors. During follow-up periods of 1-11 months, 10 patients (19.2\%) remained cancer-free after TACE. Conclusion: Tracking navigation imaging with 3D cone-beam CT angiography should be useful for TACE in HCC patients with complicated feeding arteries.

Copyright (c) 2014 S. Karger AG, Basel

\section{Introduction}

Transcatheter arterial chemoembolization (TACE) is used widely in the treatment of hepatocellular carcinomas (HCC) that are not amenable to surgical resection or to percutaneous ablation therapies [1-8]. Usually, visualization of the liver arterial branch during TACE is guided by two-dimensional (2D) angiography [9-11]. Detailed information of the feeding vessels of HCC is essential for successful catheterization and sufficient TACE [7, 12]. However, it is sometimes difficult to identify some arteries feeding HCCs on 2D angiographic images because of overlapping vessels. CT during hepatic arteriography is very useful for detecting hypervascular HCC and identifying the feeding vessels [13-15]. The unified angiography/CT system allows angiography and CT to be conducted with the patient on the same bed, thus minimizing the risk of catheter dislodgment $[16,17]$. However, such systems are only found in a limited number of hospitals because they are rather expensive and take up a great deal of space. In many hospitals, patients must be transferred from the angiography room to a separate CT room for CT hepatic angiography.

Recently, three-dimensional (3D) cone-beam CT technology using a flat-panel detector has provided good 3D angiography and cross-sectional soft tissue imaging as party of an angiography system [18]. Cone-beam CT is a technique that permits assessment of the complex vascular anatomy of the liver [19-22], and, compared with a unified angiography/CT system, cone-beam CT is inexpensive and does not require a great deal of space. Several studies have shown that cone-beam CT angiography can be helpful during TACE, especially in patients with complex hepatic arterial anatomy [23-25]. In addition, recent software developments have enabled automatic identification of the feeding vessels on 3D angiography. The TACE procedure can be carried out with less chance of going astray in patients with complicated vessels of the liver by virtually color-coding the feeding vessels. The purpose of this study was to evaluate the usefulness of new tracking navigation imaging software for cone-beam CT angiography in patients with HCC undergoing treatment with TACE.

\section{Materials and Methods}

\section{Patients}

Approval for this retrospective study was obtained from the local ethical review board. Between May 2011 and April 2012, 52 patients with 73 HCCs were enrolled in this retrospective study. The patient population included 34 men and 18 women (age range, 54-90 years; mean \pm SD, $73.9 \pm 7.9$ years). The maximal diameter of the tumors ranged from 1.5 to $10 \mathrm{~cm}$ (mean $\pm \mathrm{SD}, 2.6 \pm 1.4 \mathrm{~cm}$ ) on dynamic CT. Thirty-six patients had liver cirrhosis of Child-Pugh class A and the remaining 16 had Child-Pugh class B cirrhosis.

All patients met the following inclusion criteria: multiple hypervascular HCCs or HCCs larger than $3 \mathrm{~cm}$ in diameter, some overlapping arteries to the tumor depicted on celiac angiography, absence of portal venous and extrahepatic metastasis, prothrombin time-international normalized ratio less than 1.7, total bilirubin less than $3.0 \mathrm{mg} / \mathrm{dl}$, and platelet count greater than $50,000 / \mu \mathrm{l}$. We diagnosed HCC based 
on the findings of three-phase contrast-enhanced CT. HCCs were positively enhanced in the arterial phase and washed out in the portal-venous and/or equilibrium phase of contrast-enhanced CT. All patients underwent contrast-enhanced CT 1 month before TACE.

\section{Cone-Beam CT and Tracking Navigation Imaging}

C-arm cone-beam CT angiography was performed using an Innova $4100^{\mathrm{IQ}}$ pro angiographic unit (GE Healthcare, Amersham, UK). Patients did not need to elevate their arms above their heads for the rotation. After breathing was suspended, there was a 5-s delay for contrast filling of the vessels and tumors prior to $\mathrm{C}$-arm rotation. Contrast medium (Omnipaque $300 \mathrm{mg} / \mathrm{ml}$; GE Healthcare) was injected at a flow rate of $1.5-2.0 \mathrm{ml} / \mathrm{s}$ through a microcatheter in the common hepatic artery, the proper hepatic artery, or the replaced hepatic artery. The total volume of contrast used for the rotation was 15-20 ml. Cone-beam CT images were acquired using the following parameters: total scanning angle, $200^{\circ}$; rotation speed, $20^{\circ}$ or $40 \%$ s; acquisition time, 5 or $10 \mathrm{~s}$; matrix size, $1500 \times 1500$; isotropic voxel size, $0.2 \mathrm{~mm}$; and effective fieldof-view, $18 \mathrm{~cm}^{2}$. During rotation, 300 images were obtained at a frame rate of 30 frames/s. Acquired images were then transferred to an external workstation (Advantage Workstation 4.2; GE Healthcare) where a volume data set was reconstructed in a CT-type data set consisting of many sections with the thickness of the voxel size and visualized with a volume-rendering technique. The reconstructed 3D field of view was $40 \times 40 \mathrm{~cm}$ and the image matrix size was $512 \times 512 \times 512$ voxels.

The tracking navigation software is an advanced vascular 3D clinical analysis tool using data from cone-beam CT angiography [26]. In the first step, we set the cross at the tip of the microcatheter on axial and coronal images. In the second step, a spherical region of interest (ROI) was adjusted around the targeted tumor on axial and coronal images. The three-dimensional positions of the starting point and the target were thereby fixed. The 3D navigation software could then automatically depict all vessels in the vicinity of the target as being feeding arteries (figs. 1,2). The vicinity was automatically determined by geometric considerations and could be displayed on the angiography room monitor in approximately 20 s after the cone-beam CT angiography procedure. This tracking navigation imaging was able to highlight all vessels (from the tip of the microcatheter to the tumor) that appeared to feed the tumor by coming into close geometric proximity with the ROI. The total time required to create the tracking navigation images per patient study was approximately $30 \mathrm{~s}$. Moreover, virtual "3D roadmap" imaging can superimpose the live fluoroscopic images with the 3D reconstruction. This 3D roadmap is automatically adjusted in real time for all changes in C-arm angulations, field of view, and table positions.

\section{The TACE Technique}

The right femoral artery was accessed with an 18-gauge Seldinger needle and a 4-Fr sheath was inserted. The celiac artery was selectively catheterized using a 4.2-Fr catheter. A 2.2-Fr microcatheter (Shirabe; Piolax, Yokohama, Japan) was advanced coaxially through the catheter into the common or proper hepatic artery. Rotational angiography was performed to evaluate the feeding vessels and to reveal hypervascular tumors in the liver. The tip of the catheter was selectively placed into the segmental and subsegmental arteries feeding the tumor in reference to selective hepatic angiography and/or the tracking navigation imaging findings. Chemoembolization was performed using 10-30 mg of epirubicin (Epirubicin; Nippon Kayaku, Tokyo, Japan) dissolved in 1-3 ml of distilled water and emulsified with $2 \mathrm{ml}$ of iodized oil (Lipiodol Ultra-Fluid; Guerbet, Paris, France) and gelatin sponge particles (Gelpart; Nippon Kayaku). The volume of the emulsion to be injected was determined by the tumor volume (maximal volume of emulsion, $8 \mathrm{ml})$.

\section{Imaging Data Evaluation and Follow-Up}

The cone-beam CT scans and the tracking navigation images were prospectively recorded and retrospectively evaluated. The primary objective of this study was, using final selective angiographic findings as the gold standard, to evaluate the diagnostic performance of the tracking navigation imaging system using 3D rotational common/proper hepatic arteriography compared with 2D celiac angiography for identifying the tumor feeding arteries. Overall, interpretation of celiac angiography, cone-beam CT, the tracking navigation imaging, and selective hepatic angiography was performed in a nonblinded manner.

The secondary objective was the TACE treatment response. The treatment response was assessed 1 or 2 months after TACE using the modified Response Evaluation Criteria in Solid Tumors (mRECIST) guideline proposed by the American Association for the Study of Liver Diseases-Journal of the National Cancer Institute [27]. If the follow-up CT images indicated successful TACE and the absence of new tumors, three-phase dynamic CT scans were repeated at 3-month intervals. Any complications were recorded. 

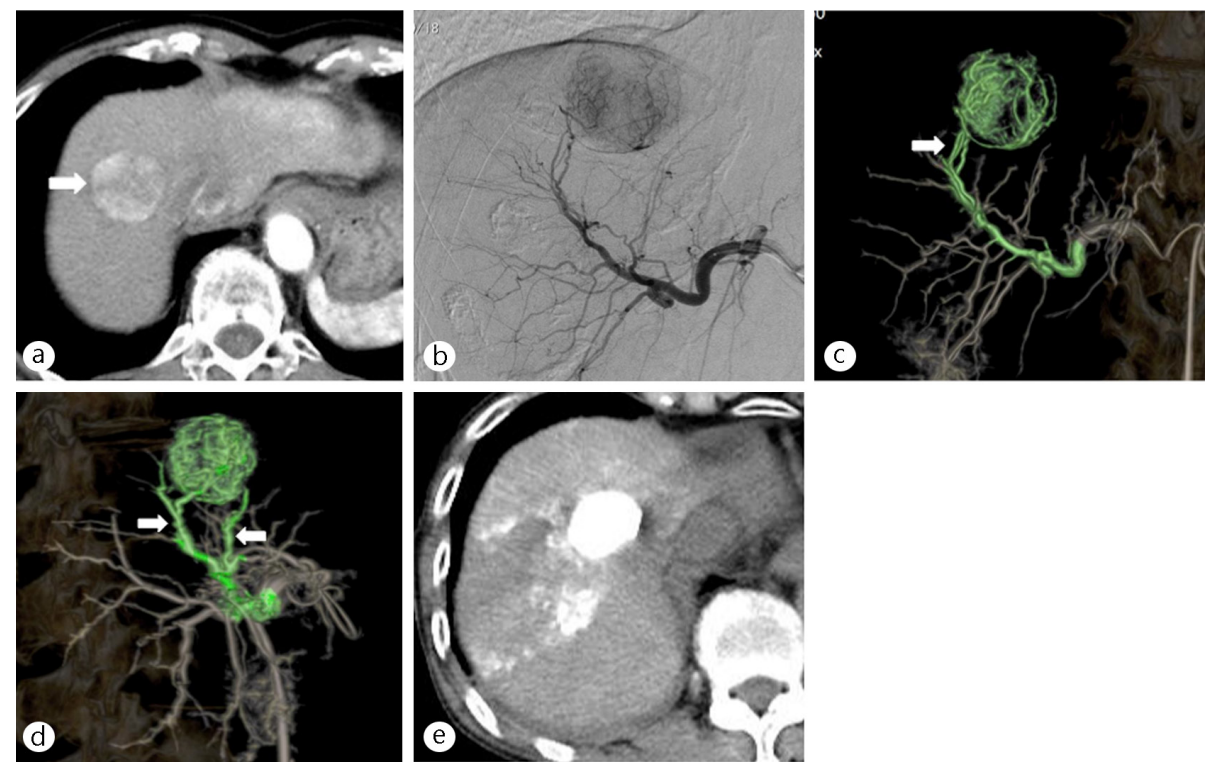

Fig. 1. The patient was a 77-year-old man with an HCC of $4.0 \mathrm{~cm}$ diameter. (a) Early-phase dynamic CT scan shows naive HCC as enhanced lesion in segment 8 of liver. (b) 2D angiography shows HCC as an enhanced lesion complicated with right anterio/posterior hepatic arteries. (c) Two main feeder vessels (arrow) are highlighted from the right anterior hepatic artery by anteroposterior view on tracking navigation imaging. (d) Two main arteries feeding from A8 (arrows) were clearly shown on the right posterior oblique view on tracking navigation imaging. (e) Plain CT scan obtained 1 month after TACE shows well-accumulated lipiodol in the tumor.
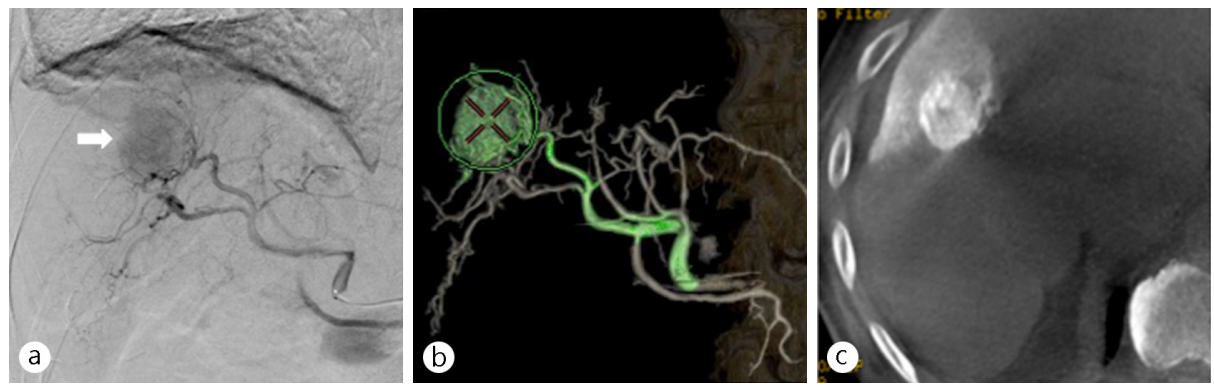

Fig. 2. The patient was a 70-year-old man with an HCC of $3.0 \mathrm{~cm}$ diameter. (a) 2D angiography shows HCC as an enhanced lesion (arrow) complicated with right anterior/posterior hepatic arteries. (b) The green vessel indicates a tumor-feeding artery from the right anterior hepatic artery to the tumor (circle) according to the tracking navigation imaging system. (c) Well-accumulated lipiodol in the tumor is shown on an axial plain 3D cone-beam CT image immediately after TACE.

\section{Statistical Analysis}

Data are expressed as mean \pm SD. Significant differences were calculated using the Wilcoxon rank sum test in detection of the feeding vessels between the tracking navigation imaging group and the celiac angiography group. A p value of less than 0.05 was considered statistically significant. The agreement of the tracking navigation imaging versus the results of selective angiography was measured using the kappa coefficient. Data were analyzed using statistical software (SPSS 11.5, Statistical Package for the Social Sciences, Chicago, IL, USA). The 95\% confidence intervals were calculated according to the binomial distribution. A kappa statistic of $0.41-0.60$ was considered to indicate moderate agreement, a weighted kappa statistic of $0.61-0.80$ was considered to indicate good agreement, and a weighted kappa statistic of 0.81-1.00 was considered to indicate excellent agreement [28]. 
Table 1. Sensitivity, specificity, positive and negative predictive values, and accuracy of 3D tracking navigation imaging and 2D celiac angiography in identifying feeder arteries of HCC

\begin{tabular}{llllll}
\hline & Sensitivity [\%] & Specificity [\%] & PPV [\%] & NPV [\%] & Accuracy [\%] \\
\hline $\begin{array}{l}\text { 3D tracking } \\
\text { navigation imaging }\end{array}$ & 97.1 & 80.0 & 98.5 & 66.7 & 90.4 \\
$\begin{array}{l}\text { 2D celiac } \\
\text { angiography }\end{array}$ & 54.4 & 60.0 & 94.9 & 8.8 & 50.7 \\
\hline
\end{tabular}

$\mathrm{NPV}=$ negative predictive value; PPV = positive predictive value .

\section{Results}

Two hundred ninety-four patients were treated by TACE or transcatheter arterial infusion chemotherapy at our hospital between May 2011 and April 2012. HCC patients who received TACE using the tracking navigation imaging system accounted for $17.7 \%(n=52)$ of the total patients treated with therapeutic angiography. Among the 73 HCC nodules in these 52 patients, 62 tumors (84.9\%) were diagnosed as naive HCCs, 8 tumors $(11.0 \%)$ as local recurrences around the lipiodol deposit, and 3 tumors (4.1\%) as residual HCCs without accumulation of lipiodol. Transfemoral TACE was carried out in 52 TACE sessions and was performed for 35 subsegmental arteries and 17 segmental arteries. Complete devascularization of postembolization angiography was achieved in all $52(100 \%)$ procedures.

The detection of feeding vessels was $90.4 \%$ (66/73) for tracking navigation imaging and $50.7 \%$ (37/73) for celiac trunk angiography. This difference was statistically significant (Wilcoxon rank sum test, $\mathrm{p}<0.001$ ). Tracking navigation imaging could not depict the feeding vessels in HCC patients with weak enhancement of tumor stain (voxel value from cone-beam CT: median 16.51; range: -59.64 to 120.76 ). However, celiac trunk angiography failed to confirm the feeding vessels not only because of weak enhancement of HCC, but also because of crossing and overlapping arteries of the liver. Using final selective angiographic findings as the gold standard, the sensitivity, specificity, positive predictive value, and negative predictive value for the detection of feeding arteries were $97.1 \%$ (66/68), 80.0\% (4/5), 98.5\% (66/67), and $66.7 \%(4 / 6)$, respectively (table 1). The kappa coefficient had a value of $0.638(95 \% \mathrm{CI}$ : 0.471-0.805).

According to mRECIST, 17 of 52 (32.7\%) individuals had a complete response, 8 (15.4\%) had a partial response, $10(19.2 \%)$ had stable disease, and 17 (32.7\%) had progressive disease. Thus, the disease control rate, defined as a complete response, partial response, or stable disease, was 67.3\%. Development of new lesions was the sole reason for progression in all patients with progressive disease. The follow-up period ranged from 1 to 11 months (mean \pm SD, $6.1 \pm 4.2$ months). During follow-up, 10 patients (19.2\%) remained cancer-free after TACE, 31 patients (59.6\%) received or were scheduled for additional TACE, 3 patients $(5.8 \%)$ received sorafenib, 3 patients (5.8\%) received radiofrequency ablation, and 5 patients $(9.6 \%)$ received best supportive care. No serious side effects or procedure-related complications (e.g., hemorrhage, infection, hepatic failure, or death) occurred. 


\section{Discussion}

Among the many techniques for 3D image manipulation, maximum intensity projection (MIP) and volume rendering are widely used for 3D vascular imaging [13-17]. MIP is a specific type of rendering in which the brightest voxel is projected into the 3D image. Unlike MIP, which displays only a small fraction of the available data, volume rendering can display a volume of data in its entirety. This property allows volume-rendered CT angiograms to provide vessel "depth," to display multiple overlapping vessels much more clearly, and to exclude surrounding structures [29-31]. Recently, the medical imaging community has embraced volume rendering for a wide variety of 3D imaging applications including navigation imaging based on cone-beam CT.

The new 3D navigation imaging software successfully traced feeding arteries in the vicinity of the target. In this study, 3D navigation imaging was found to be significantly better for accurate detection of feeding arteries than 2D celiac angiography was $(p<0.001)$. A good degree of agreement was achieved between 3D navigation imaging and the final angiographic findings (kappa $=0.638$ ). Our results indicate that this tracking navigation imaging system could contribute to selection of the feeding arteries and could assist with therapeutic angiography for unresectable HCC, especially in patients with complicated feeding anatomies. In the 52 patients who underwent TACE using the tracking navigation imaging system, the disease control rate by TACE was $67.3 \%$, according to selective angiography. During followup (mean, 6.1 months; range, 1-11 months), 19.2\% remained recurrence-free in this study.

This new tracking navigation imaging system with cone-beam CT has three important features for clinical application as part of TACE. The first is speed. The tracking image can be displayed on the angiography room monitor approximately $30 \mathrm{~s}$ after completion of the cone-beam CT angiography procedure, and the operator can get feedback to advance the catheter. The second feature is high resolution: clear images of intrahepatic arterial branches can be obtained without patients having to raise their arms. The third feature is multifunctionality. Not only a 3D rotational view but also a 3D roadmap can help the operator position the catheter using less $x$-ray dye and in a shorter time. Modern vessel identification software that uses cone-beam CT data has been developed. Wang et al. reported that the 3D vessel tracking system has advantages over conventional 2D hepatic angiography in revealing the cystic artery $(p<0.001)$ [32]. Miyayama et al. found the usefulness of automatic feeder vessel detection software with a true-positive ratio with $88 \%$ in the identification of the tumor-feeding artery [33]. Iwazawa et al. also showed that the sensitivity in detecting tumor feeders was $87.7 \%$ [34]. They reported that the software improved the sensitivity of tumor feeder detection with a shorter processing time than manual assessment angiography.

In the present study, in four HCCs in four patients, neither this tracking imaging method nor selective angiography could display the feeding arteries; this shortcoming might be the result of poor enhancement of the feeding arteries and tumors. Excess feeding vessels were depicted in a HCC in one patient. This error might be the result of high sensitivity with rich enhancement of huge HCCs. In one patient, 3D tracking navigation imaging failed to detect the inferior phrenic artery feeding the HCC. Finally, selective angiography could correct false-positive detection of the feeders with the software. In studies of vessel identification software use, aberrant path formation during the software extraction process was found to be caused by crossed tumor feeders, feeder vessels with hairpin turns, artifacts, and arteroportal shunts, among other complicating factors.

It is anticipated that tracking navigation imaging software using cone-beam CT will reduce radiation exposure, shorten the overall procedure time, and cut the amount of contrast material used because the feedback that it provides will facilitate efficient angiographic procedures. However, to minimize radiation exposure, we should refrain from unnecessary an- 
giography or cone-beam CT examination. Radiation exposure ranges from 15 to $20 \mathrm{mSv}$ for an abdominal angiogram, and the effective dose is $4-5 \mathrm{mSv}$ for a cone-beam CT abdominal scan $[35,36]$. The need for clinical imaging information must be balanced against the potential negative effects of radiation.

The principal limitation of this study was its retrospective design, which inherently decreased the statistical strength. The second was that this study could suffer from selection bias because the patients were enrolled according to the operators' subjective judgment. However, tracking navigation imaging could assist therapeutic angiography and contribute to the effectiveness of TACE for HCC despite the inclusion of patients with complicated feeding vessels. The third limitation was the preliminary nature of this study with a relatively small number of patients. Further studies of this technique with a larger number of patients are warranted.

In summary, tracking navigation imaging with 3D cone-beam CT angiography can provide immediate feedback on the angiographic procedure and could easily identify HCC tumor feeders. Thereafter, these feeders could be catheterized quickly with the patient receiving low contrast/radiation doses. This new 3D tracking navigation imaging software can inspire greater confidence and should be useful for TACE in HCC patients with complicated feeding arteries.

\section{Author Contributions}

Yasunori Minami was responsible for the acquisition of clinical data and the writing of the manuscript. Takamichi Murakami helped develop the new tracking navigation imaging system and edited the manuscript. Yukinobu Yagyu provided technical instruction on 3D cone-beam CT angiography. Masatoshi Kudo reviewed and approved the final version of the manuscript.

\section{Conflict of Interest}

Y.M. Financial activities related to the present article: none to disclose. Financial activities not related to the present article: none to disclose. Other relationships: none to disclose. M.K. Financial activities related to the present article: none to disclose. Financial activities not related to the present article: none to disclose. Other relationships: none to disclose. Y.Y. Financial activities related to the present article: none to disclose. Financial activities not related to the present article: none to disclose. Other relationships: none to disclose. T.M. Financial activities related to the present article: none to disclose. Financial activities not related to the present article: none to disclose. Other relationships: none to disclose.

\section{References}

1 Nakamura H, Hashimoto T, Oi H, Sawada S: Transcatheter oily chemoembolization of hepatocellular carcinoma. Radiology 1989;170:783-786.

-2 Matsui O, Kadoya M, Yoshikawa J, Gabata T, Arai K, Demachi H, Miyayama S, Takashima T, Unoura M, Kogayashi K: Small hepatocellular carcinoma: treatment with subsegmental transcatheter arterial embolization. Radiology 1993;188:79-83.

-3 Takayasu K, Arii S, Ikai I, Omata M, Okita K, Ichida T, Matsuyama Y, Nakanuma Y, Kojiro M, Makuuchi M, Yamaoka Y, Liver Cancer Study Group of Japan: Prospective cohort study of transarterial chemoembolization for unresectable hepatocellular carcinoma in 8510 patients. Gastroenterology 2006;131:461-469.

4 Llovet JM, Real MI, Montaña X, Planas R, Coll S, Aponte J, Ayuso C, Sala M, Muchart J, Solà R, Rodés J, Bruix J, Barcelona Liver Cancer Group: Arterial embolisation or chemoembolisation versus symptomatic treatment in patients with unresectable hepatocellular carcinoma: a randomised controlled trial. Lancet 2002;359:1734-1739. 
5 Sergio A, Cristofori C, Cardin R, Pivetta G, Ragazzi R, Baldan A, Girardi L, Cillo U, Burra P, Giacomin A, Farinati F: Transcatheter arterial chemoembolization (TACE) in hepatocellular carcinoma (HCC): the role of angiogenesis and invasiveness. Am J Gastroenterol 2008;103:914-921.

-6 Imai Y, Chikayama T, Nakazawa M, Watanabe K, Ando S, Mizuno Y, Yoshino K, Sugawara K, Hamaoka K, Fujimori K, Inao M, Nakayama N, Oka M, Nagoshi S, Mochida S: Usefulness of miriplatin as an anticancer agent for transcatheter arterial chemoembolization in patients with unresectable hepatocellular carcinoma. J Gastroenterol 2011;47:179-186.

7 Lencioni R: Chemoembolization in patients with hepatocellular carcinoma. Liver Cancer 2012;1:41-50.

-8 Ricke J, Seidensticker M, Mohnike K: Noninvasive diagnosis of hepatocellular carcinoma in cirrhotic liver: current guidelines and future prospects for radiological imaging. Liver Cancer 2012;1:51-58.

-9 Lewandowski RJ, Geschwind JF, Liapi E, Salem R: Transcatheter intraarterial therapies: rationale and overview. Radiology 2011;259:641-657.

10 Lencioni R, Crocetti L: Local-regional treatment of hepatocellular carcinoma. Radiology 2012;262:43-58.

-11 Bouvier A, Ozenne V, Aubé C, Boursier J, Vullierme MP, Thouveny F, Farges O, Vilgrain V: Transarterial chemoembolisation: effect of selectivity on tolerance, tumour response and survival. Eur Radiol 2011;21:1719-1726.

12 Gaba RC: Chemoembolization practice patterns and technical methods among interventional radiologists: results of an online survey. AJR Am J Roentgenol 2012;198:692-699.

13 Nelson RC, Chezmar JL, Sugarbaker PH, Bernardino ME: Hepatic tumors: comparison of CT during arterial portography, delayed CT, and MR imaging for preoperative evaluation. Radiology 1989;172:27-34.

14 Kanematsu M, Hoshi H, Imaeda T, Murakami T, Inaba Y, Yokoyama R, Nakamura H: Detection and characterization of hepatic tumors: value of combined helical CT hepatic arteriography and CT during arterial portography. AJR Am J Roentgenol 1997;168:1193-1198.

15 Murakami T, Oi H, Hori M, Kim T, Takahashi S, Tomoda K, Narumi Y, Nakamura H: Helical CT during arterial portography and hepatic arteriography for detecting hypervascular hepatocellular carcinoma. AJR Am J Roentgenol 1997;169:131-135.

16 Takayasu K, Muramatsu Y, Maeda T, Iwata R, Furukawa H, Muramatsu Y, Moriyama N, Okusaka T, Okada $\mathrm{S}$, Ueno H: Targeted transarterial oily chemoembolization for small foci of hepatocellular carcinoma using a unified helical CT and angiography system: analysis of factors affecting local recurrence and survival rates. AJR Am J Roentgenol 2001;176:681-688.

$\checkmark 17$ Toyoda H, Kumada T, Sone Y: Impact of a unified CT angiography system on outcome of patients with hepatocellular carcinoma. AJR Am J Roentgenol 2009;192:766-774.

18 Lee JM, Yoon JH, Joo I, Woo HS: Recent advances in CT and MR imaging for evaluation of hepatocellular carcinoma. Liver Cancer 2012;1:22-40.

-19 Hirota S, Nakao N, Yamamoto S, Kobayashi K, Maeda H, Ishikura R, Miura K, Sakamoto K, Ueda K, Baba R: Cone-beam CT with flat-panel-detector digital angiography system: early experience in abdominal interventional procedures. Cardiovasc Intervent Radiol 2006;29:1034-1038.

20 Kakeda S, Korogi Y, Ohnari N, Moriya J, Oda N, Nishino K, Miyamoto W: Usefulness of cone-beam volume CT with flat panel detectors in conjunction with catheter angiography for transcatheter arterial embolization. J Vasc Interv Radiol 2007;18:1508-1516.

21 Loffroy R, Lin M, Rao P, Bhagat N, Noordhoek N, Radaelli A, Blijd J, Geschwind JF: Comparing the detectability of hepatocellular carcinoma by $\mathrm{C}$-arm dual-phase cone-beam computed tomography during hepatic arteriography with conventional contrast-enhanced magnetic resonance imaging. Cardiovasc Intervent Radiol 2012;35:97-104.

-22 Miyayama S, Yamashiro M, Hashimoto M, Hashimoto N, Ikuno M, Okumura K, Yoshida M, Matsui O: Blood supply of the main bile duct from the caudate artery and medial subsegmental artery of the hepatic artery: Evaluation using images obtained during transcatheter arterial chemoembolization for hepatocellular carcinoma. Hepatol Res 2013. [Epub ahead of print].

23 Miyayama S, Yamashiro M, Okuda M, Yoshie Y, Sugimori N, Igarashi S, Nakashima Y, Matsui O: Usefulness of cone-beam computed tomography during ultraselective transcatheter arterial chemoembolization for small hepatocellular carcinomas that cannot be demonstrated on angiography. Cardiovasc Intervent Radiol 2009;32:255-264.

24 Miyayama S, Yamashiro M, Hattori Y, Orito N, Matsui K, Tsuji K, Yoshida M, Matsui O: Efficacy of conebeam computed tomography during transcatheter arterial chemoembolization for hepatocellular carcinoma. Jpn J Radiol 2011;29:371-377.

25 Higashihara H, Osuga K, Onishi H, Nakamoto A, Tsuboyama T, Maeda N, Hori M, Kim T, Tomiyama N: Diagnostic accuracy of $\mathrm{C}$-arm CT during selective transcatheter angiography for hepatocellular carcinoma: comparison with intravenous contrast-enhanced, biphasic, dynamic MDCT. Eur Radiol 2012;22:872-879.

-26 Deschamps F, Solomon SB, Thornton RH, Rao P, Hakime A, Kuoch V, de Baere T: Computed analysis of three-dimensional cone-beam computed tomography angiography for determination of tumor-feeding vessels during chemoembolization of liver tumor: a pilot study. Cardiovasc Intervent Radiol 2010. [Epub ahead of print].

27 Lencioni R, Llovet JM: Modified RECIST (mRECIST) assessment for hepatocellular carcinoma. Semin Liver Dis 2010;30:52-60.

28 Landis JT, Koch GG: The measurement of observer agreement for categorical data.Biometrics 1977;13:159174.

-29 Calhoun PS, Kuszyk BS, Heath DG, Carley JC, Fishman EK: Three-dimensional volume rendering of spiral CT data: theory and method. Radiographics 1999;19:745-764. 
30 Dalrymple NC, Prasad SR, Freckleton MW, Chintapalli KN: Informatics in radiology (infoRAD): introduction to the language of three-dimensional imaging with multidetector CT. Radiographics 2005;25:14091428.

31 Fishman EK, Ney DR, Heath DG, Corl FM, Horton KM, Johnson PT: Volume rendering versus maximum intensity projection in CT angiography: what works best, when, and why. Radiographics 2006;26:905-922.

-32 Wang X, Shah RP, Maybody M, Brown KT, Getrajdman GI, Stevenson C, Petre EN, Solomon SB: Cystic artery localization with a three-dimensional angiography vessel tracking system compared with conventional two-dimensional angiography. J Vasc Interv Radiol 2011;22:1414-1419.

-33 Miyayama S, Yamashiro M, Hashimoto M, Hashimoto N, Ikuno M, Okumura K, Yoshida M, Matsui O: Identification of small hepatocellular carcinoma and tumor-feeding branches with cone-beam CT guidance technology during transcatheter arterial chemoembolization. J Vasc Interv Radiol 2013;24:501-508.

34 Iwazawa J, Ohue S, Hashimoto N, Muramoto O, Mitani T: Clinical utility and limitations of tumor-feeder detection software for liver cancer embolization. Eur J Radiol 2013;82:1665-1671.

35 Koyama S, Aoyama T, Oda N, Yamauchi-Kawaura C: Radiation dose evaluation in tomosynthesis and C-arm cone-beam CT examinations with an anthropomorphic phantom. Med Phys 2010;37:4298-4306.

36 Verdun FR, Bochud F, Gundinchet F, Aroua A, Schnyder P, Meuli R: Quality initiatives* radiation risk: what you should know to tell your patient. Radiographics 2008;28:1807-1816. 